\title{
Some results on fixed points of nonlinear operators and solutions of equilibrium problems
}

Peng Cheng ${ }^{\mathrm{a}, *}$, Zhaocui Min ${ }^{\mathrm{b}}$

${ }^{a}$ School of Mathematics and Information Science, North China University of Water Resources and Electric Power, Henan, China.

${ }^{b}$ School of Science, Hebei University of Engineering, Hebei, China.

Communicated by Y. J. Cho

\begin{abstract}
The purpose of this paper is to investigate fixed points of an asymptotically quasi- $\phi$-nonexpansive mapping in the intermediate sense and a bifunction equilibrium problem. We obtain a strong convergence theorem of solutions in the framework of Banach spaces. (c)2016 All rights reserved.
\end{abstract}

Keywords: Asymptotically quasi- $\phi$-nonexpansive mapping, equilibrium problem, fixed point, variational inequality, iterative process.

2010 MSC: 65J15, 65K10.

\section{Introduction and Preliminaries}

Let $E$ be a real Banach space and let $C$ be a convex closed subset of $E$. Let $B: C \times C \rightarrow \mathbb{R}$, where $\mathbb{R}$ denotes the set of real numbers, be a bifunction. Recall that the following equilibrium problem in the terminology of Blum and Oettli [4]. Find $\bar{x} \in C$ such that

$$
B(\bar{x} y) \geq 0, \forall y \in C \text {. }
$$

In this paper, we use $\operatorname{Sol}(B)$ to denote the solution set of equilibrium problem (1.1). That is, $\operatorname{Sol}(B)=$ $\{x \in C: B(x, y) \geq 0, \forall y \in C\}$.

The following restrictions on bifunction $B$ are essential in this paper.

(Q1) $B(a, a) \equiv 0, \forall a \in C$;

\footnotetext{
* Corresponding author

Email address: hschengp@yeah.net (Peng Cheng)
} 
(Q2) $B(b, a)+B(a, b) \leq 0, \forall a, b \in C$;

(Q3) $B(a, b) \geq \lim \sup _{t \rightarrow 0} B(t c+(1-t) a, b), \forall a, b, c \in C$;

(Q4) $b \mapsto B(a, b)$ is weakly lower semi-continuous and convex, $\forall a \in C$.

Equilibrium problem (1.1), which includes complementarity problems, variational inequality problems and inclusion problems as special cases, provides us a natural and unified framework to study a wide class of problems arising in physics, economics, finance, transportation, network, elasticity and optimization; see [3], [8], 10], [12], [14], 23], 28, and the references therein. Recently, equilibrium problem (1.1) has been extensively investigated based on fixed point algorithms in Banach spaces; see [9], [11, [13], [15]-[18], [24]-27], 29]-32] and the references therein.

Let $E^{*}$ be the dual space of $E$. Let $S^{E}$ be the unit sphere of $E$. Recall that $E$ is said to be a strictly convex space iff $\|x+y\|<2$ for all $x, y \in S^{E}$ and $x \neq y$. Recall that $E$ is said to have a Gâteaux differentiable norm iff $\lim _{t \rightarrow 0} \frac{1}{t}(\|x\|-\|x+t y\|)$ exists for each $x, y \in S^{E}$. In this case, we also say that $E$ is smooth. $E$ is said to have a uniformly Gâteaux differentiable norm if for each $y \in B_{E}$, the limit is attained uniformly for all $x \in S^{E}$. $E$ is also said to have a uniformly Fréchet differentiable norm iff the above limit is attained uniformly for $x, y \in S^{E}$. In this case, we say that $E$ is uniformly smooth.

Recall that the normalized duality mapping $J$ from $E$ to $2^{E^{*}}$ is defined by

$$
J x=\left\{y \in E^{*}:\|x\|^{2}=\langle x, y\rangle=\|y\|^{2}\right\} .
$$

It is known

if $E$ is uniformly smooth, then $J$ is uniformly norm-to-norm continuous on every bounded subset of $E$;

if $E$ is a strictly convex Banach space, then $J$ is strictly monotone;

if $E$ is a smooth Banach space, then $J$ is single-valued and demicontinuous, i.e., continuous from the strong topology of $E$ to the weak star topology of $E$;

if $E$ is a reflexive and strictly convex Banach space with a strictly convex dual $E^{*}$ and $J^{*}: E^{*} \rightarrow E$ is the normalized duality mapping in $E^{*}$, then $J^{-1}=J^{*}$;

if $E$ is a smooth, strictly convex and reflexive Banach space, then $J$ is single-valued, one-to-one and onto.

From now on, we use $\rightarrow$ and $\rightarrow$ to stand for the weak convergence and strong convergence, respectively. Recall that $E$ is said to have the Kadec-Klee property (KK property) if $\lim _{n \rightarrow \infty}\left\|x_{n}-x\right\|=0$ as $n \rightarrow \infty$, for any sequence $\left\{x_{n}\right\} \subset E$, and $x \in E$ with $x_{n} \rightarrow x$, and $\left\|x_{n}\right\| \rightarrow\|x\|$ as $n \rightarrow \infty$.

Let $T$ be a mapping on $C$. Recall that a point $p$ is said to be a fixed point of $T$ if and only if $p=T p$. $p$ is said to be an asymptotic fixed point [22] of $T$ if and only if $C$ contains a sequence $\left\{x_{n}\right\}$, where $x_{n} \rightarrow p$ such that $x_{n}-T x_{n} \rightarrow 0$. From now on, we use Fix $(T)$ to stand for the fixed point set and $\widetilde{F i x}(T)$ to stand for the asymptotic fixed point set.

Next, we assume that $E$ is a smooth Banach space which means $J$ is single-valued. Study the functional

$$
\phi(x, y):=\|x\|^{2}+\|y\|^{2}-2\langle x, J y\rangle, \quad \forall x, y \in E .
$$

Let $C$ be a closed convex subset of a real Hilbert space $H$. For any $x \in H$, there exists a unique nearest point in $C$, denoted by $P_{C} x$, such that $\left\|x-P_{C} x\right\| \leq\|x-y\|$, for all $y \in C$. The operator $P_{C}$ is called the metric projection from $H$ onto $C$. It is known that $P_{C}$ is firmly nonexpansive. In [2], Alber studied a new mapping $\operatorname{Proj}_{C}$ in a Banach space $E$ which is an analogue of $P_{C}$, the metric projection, in Hilbert spaces. Recall that the generalized projection $\operatorname{Proj}_{C}: E \rightarrow C$ is a mapping that assigns to an arbitrary point $x \in E$ the minimum point of $\phi(x, y)$, which implies from the definition of $\phi$ that

$$
(\|y\|+\|x\|)^{2} \geq \phi(x, y) \geq(\|x\|-\|y\|)^{2}, \quad \forall x, y \in E .
$$

Recall that $T$ is said to be relatively nonexpansive [6], [7] iff

$$
F i x(T)=\widetilde{F i x}(T) \neq \emptyset, \phi(p, T x) \leq \phi(p, x), \quad \forall x \in C, \forall p \in F i x(T) .
$$


$T$ is said to be relatively asymptotically nonexpansive [1] iff

$$
\operatorname{Fix}(T)=\widetilde{F i x}(T) \neq \emptyset, \phi\left(p, T^{n} x\right) \leq\left(\mu_{n}+1\right) \phi(p, x), \quad \forall x \in C, \forall p \in \operatorname{Fix}(T), \forall n \geq 1,
$$

where $\left\{\mu_{n}\right\} \subset[0, \infty)$ is a sequence such that $\mu_{n} \rightarrow 0$ as $n \rightarrow \infty$.

$T$ is said to be relatively asymptotically nonexpansive in the intermediate sense iff $F i x(T)=\widetilde{F i x}(T) \neq \emptyset$ and

$$
\limsup _{n \rightarrow \infty} \sup _{p \in \operatorname{Fix}(T), x \in C}\left(\phi\left(p, T^{n} x\right)-\phi(p, x)\right) \leq 0 .
$$

Putting $\xi_{n}=\max \left\{0, \sup _{p \in F i x(T), x \in C}\left(\phi\left(p, T^{n} x\right)-\phi(p, x)\right)\right\}$, we see $\xi_{n} \rightarrow 0$ as $n \rightarrow \infty$.

$T$ is said to be quasi- $\phi$-nonexpansive [19] iff

$$
F i x(T) \neq \emptyset, \phi(p, T x) \leq \phi(p, x), \quad \forall x \in C, \forall p \in F i x(T) .
$$

$T$ is said to be asymptotically quasi- $\phi$-nonexpansive [20] iff there exists a sequence $\left\{\mu_{n}\right\} \subset[0, \infty)$ with $\mu_{n} \rightarrow 0$ as $n \rightarrow \infty$ such that

$$
F i x(T) \neq \emptyset, \phi\left(p, T^{n} x\right) \leq\left(\mu_{n}+1\right) \phi(p, x), \quad \forall x \in C, \forall p \in F i x(T), \forall n \geq 1 .
$$

$T$ is said to be asymptotically quasi- $\phi$-nonexpansive in the intermediate sense [21] iff $F i x(T) \neq \emptyset$ and

$$
\limsup _{n \rightarrow \infty} \sup _{p \in F i x(T), x \in C}\left(\phi\left(p, T^{n} x\right)-\phi(p, x)\right) \leq 0 .
$$

Putting $\xi_{n}=\max \left\{0, \sup _{p \in F i x(T), x \in C}\left(\phi\left(p, T^{n} x\right)-\phi(p, x)\right)\right\}$, we see $\xi_{n} \rightarrow 0$ as $n \rightarrow \infty$.

Remark 1.1. The class of relatively asymptotically nonexpansive mappings covers the class of relatively nonexpansive mappings. The class of (asymptotically) quasi- $\phi$-nonexpansive mappings (in the intermediate sense) is more desirable than the class of relatively (asymptotically) nonexpansive mappings (in the intermediate sense) because of restriction $\operatorname{Fix}(T)=\widetilde{F i x}(T)$.

Remark 1.2. The class of asymptotically quasi- $\phi$-nonexpansive mappings in the intermediate sense is reduced to the class of asymptotically quasi-nonexpansive mappings in the intermediate sense, which was considered in [5] as a non-Lipschitz continuous mappings, in the framework of Hilbert spaces.

Lemma 1.3 ([2]). Let $E$ be a strictly convex, reflexive, and smooth Banach space and let $C$ be a closed and convex subset of $E$. Let $x \in E$. Then

$$
\phi(y, x)-\phi\left(\Pi_{C} x, x\right) \geq \phi\left(y, \Pi_{C} x\right), \quad \forall y \in C,
$$

$\left\langle y-x_{0}, J x-J x_{0}\right\rangle \leq 0, \forall y \in C$ if and only if $x_{0}=\Pi_{C} x$.

Lemma 1.4 ([24]). Let $E$ be a strictly convex, smooth, and reflexive Banach space and let $C$ be a closed convex subset of $E$. Let $B$ be a function with restrictions (Q1), (Q2), (Q3) and (Q4). Let $x \in E$ and let $r>0$. Then there exists $z \in C$ such that $r B(z, y)+\langle z-y, J z-J x\rangle \leq 0, \forall y \in C$ Define a mapping $W^{B, r}$ by

$$
W^{B, r} x=\{z \in C: r B(z, y)+\langle y-z, J z-J x\rangle \geq 0, \quad \forall y \in C\} .
$$

The following conclusions hold:

(1) $W^{B, r}$ is single-valued quasi- $\phi$-nonexpansive.

(2) $\operatorname{Sol}(B)=F i x\left(W^{B, r}\right)$ is closed and convex.

Lemma 1.5 ([21]). Let $E$ be a strictly convex, smooth and reflexive Banach space such that both $E^{*}$ and $E$ have the KK property. Let $C$ be a convex and closed subset of $E$ and let $T$ be an asymptotically quasi- $\phi$ nonexpansive mapping in the intermediate sense on $C$. Then Fix $(T)$ is convex. 


\section{Main results}

Theorem 2.1. Let $E$ be a smooth, strictly convex, and reflexive Banach space such that both $E$ and $E^{*}$ have the $K K$ property and let $C$ be a convex and closed subset of $E$. Let $B$ be a bifunction satisfying (Q1), (Q2), (Q3) and (Q4) and let $T$ be an asymptotically quasi- $\phi$-nonexpansive mapping in the intermediate sense on $C$. Assume that $T$ is uniformly asymptotically regular and closed and Fix $(T) \cap \operatorname{Sol}(B) \neq \emptyset$. Let $\left\{x_{n}\right\}$ be a sequence generated by

$$
\left\{\begin{array}{l}
x_{0} \in E \text { chosen arbitrarily, } \\
C_{1}=C, x_{1}=\operatorname{Proj}_{C_{1}} x_{0} \\
r_{n} B\left(u_{n}, \mu\right) \geq\left\langle u_{n}-\mu, J u_{n}-J x_{n}\right\rangle, \mu \in C, \\
J y_{n}=\alpha_{n} J T^{n} u_{n}+\left(1-\alpha_{n}\right) J x_{n}, \\
C_{n+1}=\left\{z \in C_{n}: \phi\left(z, x_{n}\right)+\xi_{n} \geq \phi\left(z, y_{n}\right)\right\} \\
x_{n+1}=\operatorname{Proj}_{C_{n+1}} x_{1},
\end{array}\right.
$$

where $\xi_{n}=\max \left\{\sup _{p \in F i x(T), x \in C}\left(\phi\left(p, T^{n} x\right)-\phi(p, x)\right), 0\right\},\left\{\alpha_{n}\right\}$ is a real sequence in $[a, 1]$, where $a \in(0,1]$ is a real number, and $\left\{r_{n}\right\} \subset[r, \infty)$ is a real sequence, where $r$ is some positive real number. Then $\left\{x_{n}\right\}$ converges strongly to $\operatorname{Proj}_{F i x(T) \cap S o l(B)} x_{1}$.

Proof. The proof is split into seven steps.

Step 1. Prove $\operatorname{Sol}(B) \cap F i x(T)$ is convex and closed.

Using Lemma 1.4 and Lemma 1.5 , we find that $\operatorname{Sol}(B)$ is convex and closed and $F i x(T)$ is convex. Since $T$ is closed, one has Fix $(T)$ is also closed. So, $\operatorname{Sol}(B) \cap F i x(T)$ is convex and closed. $\operatorname{Proj}_{\operatorname{Sol}(B) \cap F i x(T)} x$ is well defined, for any element $x$ in $E$.

Step 2. Prove $C_{n}$ is convex and closed.

It is obvious that $C_{1}=C$ is convex and closed. Assume that $C_{m}$ is convex and closed for some $m \geq 1$. Let $p_{1}, p_{2} \in C_{m+1}$. It follows that $p=s p_{1}+(1-s) p_{2} \in C_{m}$, where $s \in(0,1)$. Notice that $\phi\left(p_{1}, y_{m}\right)-\phi\left(p_{1}, x_{m}\right) \leq \xi_{m}$, and $\phi\left(p_{2}, y_{m}\right)-\phi\left(p_{2}, x_{m}\right) \leq \xi_{m}$. Hence, one has

$$
\xi_{m}+\left\|x_{m}\right\|^{2}-\left\|y_{m}\right\|^{2} \geq 2\left\langle p_{1}, J x_{m}-J y_{m}\right\rangle
$$

and

$$
\xi_{m}+\left\|x_{m}\right\|^{2}-\left\|y_{m}\right\|^{2} \geq 2\left\langle p_{2}, J x_{m}-J y_{m}\right\rangle .
$$

Using the above two inequalities, one has $\phi\left(p, x_{m}\right)+\xi_{m} \geq \phi\left(z, y_{m}\right)$. This shows that $C_{m+1}$ is closed and convex. Hence, $C_{n}$ is a convex and closed set. This proves that $\operatorname{Proj}_{C_{n+1}} x_{1}$ is well defined.

Step 3. Prove $\operatorname{Sol}(B) \cap F i x(T) \subset C_{n}$.

Note that $\operatorname{Sol}(B) \cap \operatorname{Fix}(T) \subset C_{1}=C$ is clear. Suppose that $\operatorname{Sol}(B) \cap \operatorname{Fix}(T) \subset C_{m}$ for some positive integer $m$. For any $w \in \operatorname{Sol}(B) \cap \operatorname{Fix}(T) \subset C_{m}$, we see that

$$
\begin{aligned}
\phi\left(w, y_{m}\right)= & \left\|\left(1-\alpha_{m}\right) J x_{m}+\alpha_{m} J T^{m} u_{m}\right\|^{2}+\|w\|^{2} \\
& -2\left\langle w,\left(1-\alpha_{m}\right) J x_{m}+\alpha_{m} J T^{m} u_{m}\right\rangle \\
\leq & \|w\|^{2}-2 \alpha_{m}\left\langle w, J T^{m} u_{m}\right\rangle-2\left(1-\alpha_{m}\right)\left\langle w, J x_{m}\right\rangle \\
& +\alpha_{m}\left\|T^{m} u_{m}\right\|^{2}+\left(1-\alpha_{m}\right)\left\|x_{m}\right\|^{2} \\
\leq & \alpha_{m} \phi\left(w, u_{m}\right)+\alpha_{m} \xi_{m}+\left(1-\alpha_{m}\right) \phi\left(w, x_{m}\right) \\
\leq & \phi\left(w, x_{m}\right)+\xi_{m},
\end{aligned}
$$

where $\xi_{m}=\max \left\{\sup _{p \in F i x(T), x \in C}\left(\phi\left(p, T^{m} x\right)-\phi(p, x)\right), 0\right\}$. This shows that $w \in C_{m+1}$. This implies that $\operatorname{Sol}(B) \cap \operatorname{Fix}(T) \subset C_{n}$.

Step 4. Prove $\left\{x_{n}\right\}$ is bounded. 
Using Lemma 1.3 , one has $\left\langle z-x_{n}, J x_{1}-J x_{n}\right\rangle \leq 0$, for any $z \in C_{n}$. It follows that

$$
0 \geq\left\langle w-x_{n}, J x_{1}-J x_{n}\right\rangle, \forall w \in \operatorname{Sol}(B) \cap F i x(T) \subset C_{n} .
$$

Using Lemma 1.3 yields that

$$
\phi\left(\Pi_{F i x(T) \cap S o l(B)} x_{1}, x_{1}\right) \geq \phi\left(x_{n}, x_{1}\right) \geq 0,
$$

which implies that $\left\{\phi\left(x_{n}, x_{1}\right)\right\}$. Hence $\left\{x_{n}\right\}$ is also a bounded sequence. Without loss of generality, we may assume $x_{n} \rightarrow \bar{x}$. Since $C_{n}$ is convex and closed, we see $\bar{x} \in C_{n}$.

Step 5. Prove $\bar{x} \in F i x(T)$.

Using the fact $\phi\left(x_{n}, x_{1}\right) \leq \phi\left(\bar{x}, x_{1}\right)$, one has

$$
\phi\left(\bar{x}, x_{1}\right) \geq \limsup _{n \rightarrow \infty} \phi\left(x_{n}, x_{1}\right) \geq \liminf _{n \rightarrow \infty} \phi\left(x_{n}, x_{1}\right)=\liminf _{n \rightarrow \infty}\left(\left\|x_{n}\right\|^{2}+\left\|x_{1}\right\|^{2}-2\left\langle x_{n}, J x_{1}\right\rangle\right) \geq \phi\left(\bar{x}, x_{1}\right) .
$$

It follows that $\lim _{n \rightarrow \infty} \phi\left(x_{n}, x_{1}\right)=\phi\left(\bar{x}, x_{1}\right)$. Hence, we have

$$
\phi\left(x_{n+1}, x_{1}\right)-\phi\left(x_{n}, x_{1}\right) \geq \phi\left(x_{n+1}, x_{n}\right) \geq 0 .
$$

Therefore, we have $\lim _{n \rightarrow \infty} \phi\left(x_{n+1}, x_{n}\right)=0$. Since $x_{n+1} \in C_{n+1}$, one sees that

$$
\phi\left(x_{n+1}, x_{n}\right)+\xi_{n} \geq \phi\left(x_{n+1}, y_{n}\right) \geq 0 .
$$

It follows that $\lim _{n \rightarrow \infty} \phi\left(x_{n+1}, y_{n}\right)=0$. Hence, one has $\lim _{n \rightarrow \infty}\left(\left\|y_{n}\right\|-\left\|x_{n+1}\right\|\right)=0$. This implies that

$$
\|\bar{x}\|=\|J \bar{x}\|=\lim _{n \rightarrow \infty}\left\|J y_{n}\right\|=\lim _{n \rightarrow \infty}\left\|y_{n}\right\| .
$$

This implies that $\left\{J y_{n}\right\}$ is bounded. Assume that $\left\{J y_{n}\right\}$ converges weakly to $y^{*} \in E^{*}$. In view of the reflexivity of $E$, we see that $J(E)=E^{*}$. This shows that there exists an element $u \in E$ such that $J y=y^{*}$. It follows that $\phi\left(x_{n+1}, y_{n}\right)+2\left\langle x_{n+1}, J y_{n}\right\rangle=\left\|x_{n+1}\right\|^{2}+\left\|J y_{n}\right\|^{2}$. Taking liminf $\operatorname{in}_{n \rightarrow \infty}$, one has $0 \geq\|\bar{x}\|^{2}-$ $2\left\langle\bar{x}, y^{*}\right\rangle+\left\|y^{*}\right\|^{2}=\|\bar{x}\|^{2}+\|J y\|^{2}-2\langle\bar{x}, J y\rangle=\phi(\bar{x}, y) \geq 0$. That is, $\bar{x}=y$, which in turn implies that $J \bar{x}=y^{*}$. Hence, $J y_{n} \rightarrow J \bar{x} \in E^{*}$. Using the KK property, we obtain $\lim _{n \rightarrow \infty} J y_{n}=J \bar{x}$. Since $J^{-1}$ is demicontinuous and $E$ has the KK property, one gets $y_{n} \rightarrow \bar{x}$, as $n \rightarrow \infty$. Using the restriction on $\left\{\alpha_{n}\right\}$, one has $\lim _{n \rightarrow \infty}\left\|J x_{n}-J T^{n} u_{n}\right\|=0$. This implies that $\lim _{n \rightarrow \infty}\left\|J T^{n} u_{n}-J \bar{x}\right\|=0$. Since $J^{-1}$ is demicontinuous, one has $T^{n} u_{n} \rightarrow \bar{x}$. Since

$$
\left\|T^{n} u_{n}\right\|-\|\bar{x}\| \mid \leq\left\|J\left(T^{n} u_{n}\right)-J \bar{x}\right\|,
$$

one has $\left\|T^{n} u_{n}\right\| \rightarrow\|\bar{x}\|$, as $n \rightarrow \infty$. Since $E$ has the KK property, we obtain $\lim _{n \rightarrow \infty}\left\|\bar{x}-T^{n} u_{n}\right\|=0$. Since $T$ is also uniformly asymptotically regular, one has $\lim _{n \rightarrow \infty}\left\|\bar{x}-T^{n+1} u_{n}\right\|=0$. That is, $T\left(T^{n} u_{n}\right) \rightarrow \bar{x}$. Using the closedness of $T$, we find $T \bar{x}=\bar{x}$. This proves $\bar{x} \in$ Fix $(T)$.

Step 6. Prove $\bar{x} \in \operatorname{Sol}(B)$.

Since $\alpha_{n} \phi\left(x_{n+1}, u_{n}\right) \leq \phi\left(x_{n+1}, x_{n}\right)+\xi_{n}$, one has $\lim _{n \rightarrow \infty} \phi\left(x_{n+1}, u_{n}\right)=0$. Hence, one has $\lim _{n \rightarrow \infty}\left(\left\|u_{n}\right\|-\right.$ $\left.\left\|x_{n+1}\right\|\right)=0$. This implies that

$$
\|\bar{x}\|=\|J \bar{x}\|=\lim _{n \rightarrow \infty}\left\|J u_{n}\right\|=\lim _{n \rightarrow \infty}\left\|u_{n}\right\|
$$

This implies that $\left\{J u_{n}\right\}$ is bounded. Assume that $\left\{J u_{n}\right\}$ converges weakly to $y^{*} \in E^{*}$. In view of the reflexivity of $E$, we see that $J(E)=E^{*}$. This shows that there exists an element $u \in E$ such that $J u=u^{*}$. It follows that

$$
\phi\left(x_{n+1}, u_{n}\right)+2\left\langle x_{n+1}, J u_{n}\right\rangle=\left\|x_{n+1}\right\|^{2}+\left\|J u_{n}\right\|^{2} .
$$

Taking $\lim \inf _{n \rightarrow \infty}$, one has

$$
0 \geq\|\bar{x}\|^{2}-2\left\langle\bar{x}, u^{*}\right\rangle+\left\|u^{*}\right\|^{2}=\|\bar{x}\|^{2}+\|J u\|^{2}-2\langle\bar{x}, J u\rangle=\phi(\bar{x}, u) \geq 0 .
$$


That is, $\bar{x}=u$, which in turn implies that $u^{*}=J \bar{x}$. Hence, $J u_{n} \rightarrow J \bar{x} \in E^{*}$. Using the KK property, we obtain $\lim _{n \rightarrow \infty} J u_{n}=J \bar{x}$. Since $J^{-1}$ is demi-continuous and $E$ has the KK property, one gets $u_{n} \rightarrow \bar{x}$, as $n \rightarrow \infty$. Since

$$
r_{n} B\left(y, u_{n}\right)+\left\langle u_{n}-y, J u_{n}-J y_{n}\right\rangle \geq 0, \forall y \in C_{n},
$$

we see that $B(y, \bar{x}) \leq 0$. Let $0<t<1$ and define $y_{t}=t y+(1-t) \bar{x}$. It follows that $y_{t} \in C$, which yields that $B\left(y_{t}, \bar{x}\right) \leq 0$. It follows from the (Q1) and (Q4) that

$$
0=B\left(y_{t}, y_{t}\right) \leq t B\left(y_{t}, y\right)+(1-t) B\left(y_{t}, \bar{x}\right) \leq t B\left(y_{t}, y\right)
$$

That is, $B\left(y_{t}, y\right) \geq 0$. It follows from (Q3) that $B(\bar{x}, y) \geq 0, \forall y \in C$. This implies that $\bar{x} \in \operatorname{Sol}(B)$. This completes the proof that $\bar{x} \in \operatorname{Sol}(B) \cap F i x(T)$.

Step 7. Prove $\bar{x}=\operatorname{Proj}_{\operatorname{Sol}(B) \cap F i x(T)} x_{1}$.

Note the fact $\left\langle w-x_{n}, J x_{1}-J x_{n}\right\rangle \leq 0, \forall w \in \operatorname{Sol}(B) \cap F i x(T)$. It follows that

$$
\left\langle\bar{x}-w, J x_{1}-J \bar{x}\right\rangle \geq 0, \quad \forall w \in F i x(T) \cap \operatorname{Sol}(B) .
$$

Using Lemma 1.3 , we find that that $\bar{x}=\operatorname{Proj}_{F i x(T) \cap S o l(B)} x_{1}$. This completes the proof.

From Theorem 2.1, the following results are not hard to derive.

Corollary 2.2. Let $E$ be a smooth, strictly convex, and reflexive Banach space such that both $E$ and $E^{*}$ have the KK property and let $C$ be a convex and closed subset of E. Let $B$ be a bifunction satisfying (Q1), (Q2), (Q3) and (Q4). Assume that $\operatorname{Sol}(B) \neq \emptyset$. Let $\left\{x_{n}\right\}$ be a sequence generated by

$$
\left\{\begin{array}{l}
x_{0} \in E \text { chosen arbitrarily, } \\
C_{1}=C, x_{1}=\operatorname{Proj}_{C_{1}} x_{0} \\
r_{n} B\left(u_{n}, \mu\right) \geq\left\langle u_{n}-\mu, J u_{n}-J x_{n}\right\rangle, \mu \in C \\
J y_{n}=\alpha_{n} J u_{n}+\left(1-\alpha_{n}\right) J x_{n} \\
C_{n+1}=\left\{z \in C_{n}: \phi\left(z, x_{n}\right) \geq \phi\left(z, y_{n}\right)\right\} \\
x_{n+1}=\operatorname{Proj}_{C_{n+1}} x_{1}
\end{array}\right.
$$

where $\left\{\alpha_{n}\right\}$ is a real sequence in $[a, 1], a \in(0,1]$ is a real number and $\left\{r_{n}\right\} \subset[r, \infty)$ is a real sequence, where $r$ is some positive real number. Then $\left\{x_{n}\right\}$ converges strongly to $\operatorname{Proj}_{\operatorname{Sol}(B)} x_{1}$.

Corollary 2.3. Let $E$ be a Hilbert space and let $C$ be a convex and closed subset of $E$. Let $B$ be a bifunction satisfying (Q1), (Q2), (Q3) and (Q4) and let $T$ be an asymptotically quasi-nonexpansive mapping in the intermediate sense on $C$. Assume that $T$ is uniformly asymptotically regular and closed and Fix $(T) \cap$ $\operatorname{Sol}(B) \neq \emptyset$. Let $\left\{x_{n}\right\}$ be a sequence generated by

$$
\left\{\begin{array}{l}
x_{0} \in E \text { chosen arbitrarily, } \\
C_{1}=C, x_{1}=P_{C_{1}} x_{0}, \\
r_{n} B\left(u_{n}, \mu\right) \geq\left\langle u_{n}-\mu, u_{n}-x_{n}\right\rangle, \mu \in C, \\
y_{n}=\alpha_{n} T^{n} u_{n}+\left(1-\alpha_{n}\right) x_{n}, \\
C_{n+1}=\left\{z \in C_{n}:\left\|z-x_{n}\right\|^{2}+\xi_{n} \geq\left\|z-y_{n}\right\|^{2}\right\} \\
x_{n+1}=P_{C_{n+1}} x_{1},
\end{array}\right.
$$

where $\xi_{n}=\max \left\{\sup _{p \in F i x(T), x \in C}\left(\left\|p-T^{n} x\right\|^{2}-\|p-x\|^{2}\right), 0\right\},\left\{\alpha_{n}\right\}$ is a real sequence in $[a, 1]$, where $a \in(0,1]$ is a real number, and $\left\{r_{n}\right\} \subset[r, \infty)$ is a real sequence, where $r$ is some positive real number. Then $\left\{x_{n}\right\}$ converges strongly to $P_{F i x(T) \cap S o l(B)} x_{1}$. 


\section{Acknowledgement}

The authors are grateful to the reviewers for the useful suggestions which improve the contents of this article.

\section{References}

[1] R. P. Agarwal, Y. J. Cho, X. Qin, Generalized projection algorithms for nonlinear operators, Numer. Funct. Anal. Optim., 28 (2007), 1197-1215.1

[2] Y. I. Alber, Metric and generalized projection operators in Banach spaces: properties and applications, in: A.G. Kartsatos (Ed.), Theory and Applications of Nonlinear Operators of Accretive and Monotone Type, Marcel Dekker, New York, (1996). 1. 1.3

[3] B. A. Bin Dehaish, X. Qin, A. Latif, H. Bakodah, Weak and strong convergence of algorithms for the sum of two accretive operators with applications, J. Nonlinear Convex Anal., 16 (2015), 1321-1336.1

[4] E. Blum, W. Oettli, From optimization and variational inequalities to equilibrium problems, Math. Student, 63 (1994), 123-145.1.

[5] R. E. Bruck, T. Kuczumow, S. Reich, Convergence of iterates of asymptotically nonexpansive mappings in Banach spaces with the uniform Opial property, Colloq. Math., 65 (1993), 169-179.1.2

[6] D. Butnariu, S. Reich, A. J. Zaslavski, Asymptotic behavior of relatively nonexpansive operators in Banach spaces, J. Appl. Anal., 7 (2001), 151-174.1

[7] Y. Censor, S. Reich, Iterations of paracontractions and firmly nonexpansive operators with applications to feasibility and optimization, Optimization, 37 (1996), 323-339.1.

[8] Y. J. Cho, J. Li, N. J. Huang, Solvability of implicit complementarity problems, Math. Comput. Model., 45 (2007), 1001-1009.1

[9] S. Y. Cho, X. Qin, On the strong convergence of an iterative process for asymptotically strict pseudocontractions and equilibrium problems, Appl. Math. Comput., 235 (2014), 430-438.1

[10] S. Y. Cho, X. Qin, L. Wang, Strong convergence of a splitting algorithm for treating monotone operators, Fixed Point Theory Appl., 2014 (2014), 15 pages.1

[11] B. S. Choudhury, S. Kundu, A viscosity type iteration by weak contraction for approximating solutions of generalized equilibrium problem, J. Nonlinear Sci. Appl., 5 (2012), 243-251.1]

[12] J. Gwinner, F. Raciti, Random equilibrium problems on networks, Math. Comput. Modelling, 43 (2006), 880-891. 1

[13] Y. Hao, Some results on a modified Mann iterative scheme in a reflexive Banach space, Fixed Point Theory Appl., 2013 (2013), 14 pages.1.

[14] R. H. He, Coincidence theorem and existence theorems of solutions for a system of Ky Fan type minimax inequalities in FC-spaces, Adv. Fixed Point Theory, 2 (2012), 47-57.1

[15] J. K. Kim, Strong convergence theorems by hybrid projection methods for equilibrium problems and fixed point problems of the asymptotically quasi- $\phi$-nonexpansive mappings, Fixed Point Theory Appl., 2011 (2011), 15 pages. 1

[16] J. K. Kim, S. Y. Cho, X. Qin, Some results on generalized equilibrium problems involving strictly pseudocontractive mappings, Acta Math. Sci., 31 (2011), 2041-2057.

[17] B. Liu, C. Zhang, Strong convergence theorems for equilibrium problems and quasi- $\phi$-nonexpansive mappings, Nonlinear Funct. Anal. Appl., 16 (2011), 365-385.

[18] R. X. Ni, J. S. Jin, C. F. Wen, Strong convergence theorems for equilibrium problems and asymptotically quasi$\phi$-nonexpansive mappings in the intermediate sense, Fixed Point Theory Appl., 2015 (2015), 23 pages. 11

[19] X. Qin, Y. J. Cho, S. M. Kang, Convergence theorems of common elements for equilibrium problems and fixed point problems in Banach spaces, J. Comput. Appl. Math., 225 (2009), 20-30.1

[20] X. Qin, Y. J. Cho, S. M. Kang, On hybrid projection methods for asymptotically quasi- $\phi$-nonexpansive mappings, Appl. Math. Comput., 215 (2010), 3874-3883.1

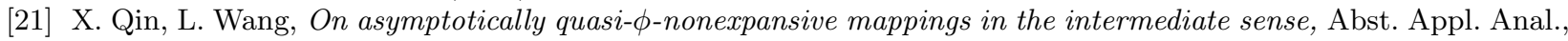
2012 (2012), 13 pages.1, 1.5

[22] S. Reich, A weak convergence theorem for the alternating method with Bregman distance, in: A.G. Kartsatos (Ed.), Theory and Applications of Nonlinear Operators of Accretive and Monotone Type, Marcel Dekker, New York, (1996). 1

[23] T. V. Su, Second-order optimality conditions for vector equilibrium problems, J. Nonlinear Funct. Anal., 2015 (2015), 31 pages.1

[24] W. Takahashi, K. Zembayashi, Strong and weak convergence theorems for equilibrium problems and relatively nonexpansive mappings in Banach spaces, Nonlinear Anal., 70 (2009), 45-57.1. 1.4

[25] Z. M. Wang, X. Zhang, Shrinking projection methods for systems of mixed variational inequalities of Browder type, systems of mixed equilibrium problems and fixed point problems, J. Nonlinear Funct. Anal., 2014 (2014), 25 pages. 
[26] Z. M. Wang, Y. Su, D. Wang, Y. Dong, A modified Halpern-type iteration algorithm for a family of hemi-relatively nonexpansive mappings and systems of equilibrium problems in Banach spaces, J. Comput. Appl. Math., 235 (2011), 2364-2371.

[27] L. Yang, F. Zhao, J. K. Kim, Hybrid projection method for generalized mixed equilibrium problem and fixed point problem of infinite family of asymptotically quasi- $\phi$-nonexpansive mappings in Banach spaces, Appl. Math. Comput., 218 (2012), 6072-6082.1

[28] Q. Yu, D. Fang, W. Du, Solving the logit-based stochastic user equilibrium problem with elastic demand based on the extended traffic network model, Eur. J. Oper. Res., 239 (2014), 112-118.1

[29] L. Zhang, H. Tong, An iterative method for nonexpansive semigroups, variational inclusions and generalized equilibrium problems, Adv. Fixed Point Theory, 4 (2014), 325-343., 1

[30] J. Zhao, S. He, Strong convergence theorems for equilibrium problems and quasi- $\phi$-asymptotically nonexpansive mappings in Banach spaces, An. St. Univ. Ovidius Constanta, 19 (2011), 347-364.

[31] J. Zhao, Approximation of solutions to an equilibrium problem in a nonuniformly smooth Banach space, J. Inequal. Appl., 2013 (2013), 10 pages.

[32] L. C. Zhao, S. S. Chang, Strong convergence theorems for equilibrium problems and fixed point problems of strict pseudo-contraction mappings, J. Nonlinear Sci. Appl., 2 (2009), 78-91.1 